\title{
Exploding and Non-exploding Stars: Coupling Nuclear Reaction Networks to Multidimensional Hydrodynamics
}

\author{
K. Kifonidis*, T. Plewa ${ }^{\dagger, *}$ and E. Müller* \\ *Max-Planck-Institut für Astrophysik, Karl-Schwarzschild-Strasse 1, D-85741 Garching, \\ Germany \\ ${ }^{\dagger}$ Nicolaus Copernicus Astronomical Center, Bartycka 18, 00716 Warsaw, Poland
}

\begin{abstract}
After decades of one-dimensional nucleosynthesis calculations, the growth of computational resources has meanwhile reached a level, which for the first time allows astrophysicists to consider performing routinely realistic multidimensional nucleosynthesis calculations in explosive and, to some extent, also in non-explosive environments. In the present contribution we attempt to give a short overview of the physical and numerical problems which are encountered in these simulations. In addition, we assess the accuracy that can be currently achieved in the computation of nucleosynthetic yields, using multidimensional simulations of core collapse supernovae as an example.
\end{abstract}

\section{INTRODUCTION}

Thermonuclear reactive flows are ubiquituous in astrophysics and occur in nonexplosive environments as, e.g., in most (hydrostatic) stars as well as in explosive events, for which novae and supernovae are examples. Often they provide the energy which powers stellar outbreaks (as in the case of novae, X-ray flashes, and thermonuclear, i.e. Type Ia, supernovae) and even for stellar explosions where this is not the case (as e.g. in core collapse supernovae, which are driven by neutrino heating), the strong coupling of hydrodynamic advection and thermonuclear reactions is of utmost importance for the nucleosynthesis which accompanies these events. It is by a proper numerical modelling of this coupling through which a more detailed insight into the origin of the nuclear abundances in the solar system can be gained, which are themselves the result of a superposition of material which has been processed in explosive and non-explosive thermonuclear environments. By comparing the results of numerical models with the observed solar abundance pattern, on the other hand, one might also hope to learn more about the thermodynamic conditions in the otherwise unaccessible nucleosynthetic sites and events themselves. 
The high precision with which nuclear abundances can be measured nowadays poses great demands on the accuracy of the numerical models, especially since it was convincingly demonstrated in recent years that due to the importance of hydrodynamic instabilities, rotation, and other effects, most of the nucleosynthetic sites do not possess spherical symmetry. Thus a reliable computation of the highly nonlinear interaction of hydrodynamic advection and nuclear burning requires multidimensional numerical models. In the following sections we give a general overview of the methods which are currently employed for modelling thermonuclear flows and discuss some of the problems which are hereby encountered. Further reviews on reactive flow modelling can be found in [9], [10] and [11].

\section{THE GOVERNING EQUATIONS}

A rather wide range of astrophysical reactive flows, in which relativistic effects, viscosity and magnetic fields can be neglected, is described by the well-known (reactive) Euler equations. This system of non-linear partial differential equations which expresses the conservation of the total mass, momentum, total (i.e. kinetic + internal) energy and baryons of the fluid reads

$$
\begin{aligned}
\frac{\partial \rho}{\partial t}+\nabla \cdot(\rho \mathbf{v}) & =0 \\
\frac{\partial \rho \mathbf{v}}{\partial t}+\nabla \cdot(\rho \mathbf{v v})+\nabla P & =\rho \mathbf{g}+\rho \mathbf{f}_{\text {add }} \\
\frac{\partial \rho E}{\partial t}+\nabla \cdot([\rho E+P] \mathbf{v}) & =\rho \mathbf{v} \cdot \mathbf{g}+\rho \dot{Q}_{\text {add }}+\rho \dot{Q}_{\text {nuc }} \\
\frac{\partial \rho X_{i}}{\partial t}+\nabla \cdot\left(\rho X_{i} \mathbf{v}\right) & =\rho \dot{X}_{i} \\
\sum_{i} X_{i} & =1,
\end{aligned}
$$

where $\rho, \mathbf{v}, E=v^{2} / 2+e$, and $P$ have their usual meanings, $X_{i}$ is the mass fraction of nucleus $i$, and $\rho \dot{X}_{i}$ as well as $\rho \dot{Q}_{\text {nuc }}$ are source terms due to nuclear transmutations. If self-gravity is important, the gravitational acceleration

$$
\mathbf{g}=-\nabla \Phi
$$

which appears in the source terms of Eqs. (2) and (3) and which depends on the gravitational potential, $\Phi$, has to be obtained from a solution of the Poisson equation

$$
\Delta \Phi=4 \pi G \rho .
$$

In mathematical terms Eqs. (1-7) describe a mixed initial/boundary value problem due to the hyperbolic and elliptic nature of the Euler and Poisson equations, respectively. Given appropriate initial and boundary conditions, an equation of 
state relating $\rho, P$ and $e$, and the additional source terms $\mathbf{f}_{\text {add }}$ and $\dot{Q}_{\text {add }}$, which in general will be problem-dependent, Eqs. (1-7) can be solved after an appropriate flow representation as well as a suitable numerical scheme have been adopted.

\section{FLOW REPRESENTATIONS AND NUMERICAL SCHEMES}

There are two primary approaches to solve the homogeneous part of the system of equations (1-5). In the Eulerian framework the system of conservation laws is solved on a grid which is fixed in space and the evolution of the flow is followed by advecting the fluid through the computational cells. The principal assets of this method are its straightforward extension from one to two or three spatial dimensions and the simplicity of its implementation on serial and parallel computer architectures. If an appropriate shock-capturing, finite-volume numerical scheme is used, it is equally straightforward to obtain strict numerical conservation of all physically conserved quantities and a sharp resolution of shocks. The major drawback is numerical diffusion. Consider the continuity equation (1) in its Eulerian form, which can be written as

$$
\frac{\partial \rho}{\partial t}+\mathbf{v} \cdot \nabla \rho+\rho \nabla \cdot \mathbf{v}=0
$$

where the second term describes advection and the third term compression. Numerical diffusion is introduced into a numerical solution of this equation as a result of discretization errors of the $\mathbf{v} \cdot \nabla$ operator. There appears to be a simple remedy to this problem: using the comoving derivative $\mathrm{d} / \mathrm{d} t=\partial / \partial t+\mathbf{v} \cdot \nabla$ we can rewrite Eq. (8) in the frame comoving with the matter to obtain its Lagrangian form

$$
\frac{\mathrm{d} \rho}{\mathrm{d} t}+\rho \nabla \cdot \mathbf{v}=0
$$

Note that in this frame the advection term $\mathbf{v} \cdot \nabla \rho$ has vanished. Therefore the Lagrangian approach is (in principle) not prone to numerical diffusion of mass (or composition). In Lagrangian methods each cell of the numerical grid represents a discretized fluid element which evolves subject to forces which are due to interactions with its neighbors and the time rate of change of the density of such a fluid element is solely determined by the compression (or expansion) that it experiences. Density interfaces (contact discontinuities) as well as composition discontinuities can be easily aligned with the boundaries of grid cells and do not have to be advected through the grid in the course of the calculation.

While this very desirable property of the Lagrangian approach has made it the method of choice for one-dimensional nucleosynthesis calculations, considerable difficulties are experienced when Lagrangian schemes are applied to multidimensional flows. Shear and vortices can severely distort a Lagrangian grid. The discrete approximation of differential operators over such a grid results in large errors in the 


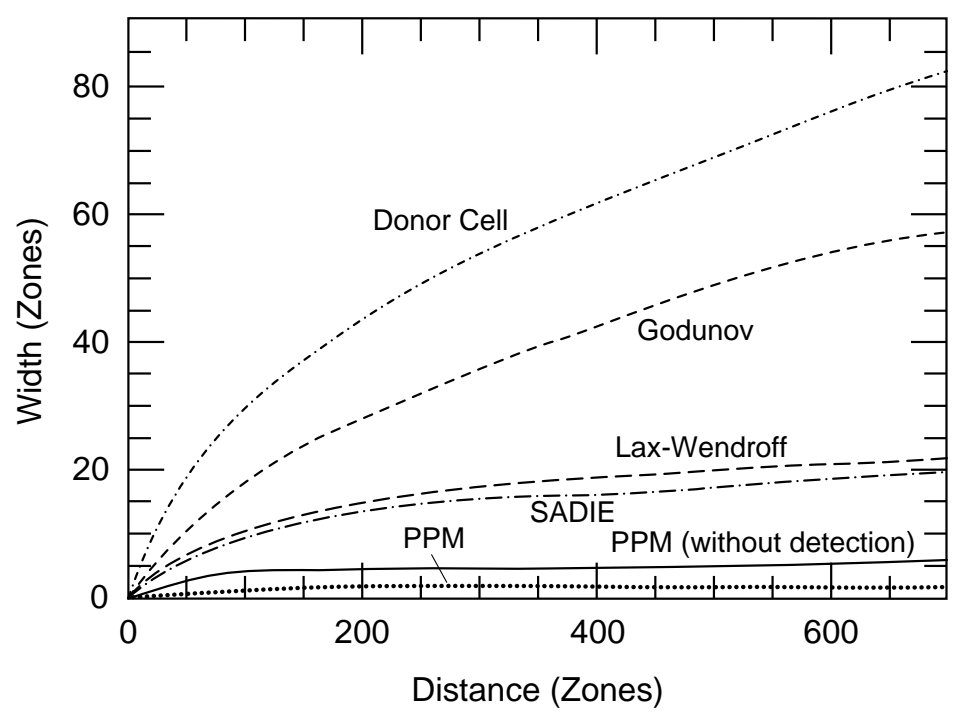

FIGURE 1. Comparison of the diffusivity of different advection schemes for the problem of the propagation of a contact discontinuity through an Eulerian grid. The curves give the width of the discontinuity (in grid zones) as a function of the number of zones it has propagated through the grid (adapted from [4] and [5]).

numerical derivatives, and in the extreme case that the grid lines cross (grid tangling) the calculations have to be stopped. Some remapping procedure to a new, more regular grid must then be applied which unavoidably introduces numerical diffusion to the solution. The distortion problem can be overcome if triangular instead of quadrilateral grids are used [11] or if (as in the Smoothed Particle Hydrodynamics, or SPH approach) no grid at all is adopted and instead the flow is sampled by a finite number of particles. In the former method considerable logic overhead is added in restructuring the deformed triangular grid, while in the latter case, due to the Monte-Carlo nature of the sampling, Poisson noise is introduced.

Due to the aforementioned drawbacks and due to significant progress in the development of accurate Eulerian schemes in the early 1980's, Lagrangian methods employing quadrilateral or triangular grids have not been used extensively in multidimensional calculations of astrophysical flows (see [18], [12] as well as [11] and the references therein for examples). On the other hand, the simplicity of SPH has made this method very popular for astrophysical (especially cosmological) simulations. Without attempting to escalate the very vigorous discussion, whether SPH or grid-based Eulerian schemes are to be prefered in astrophysical calculations (see e.g. [10]), we will argue below that, due to its Monte-Carlo nature, the SPH scheme appears to be rather unsuited for multidimensional nucleosynthesis calculations, especially in cases where hydrodynamic instabilities are known to be important.

Among Eulerian schemes, the so-called shock-capturing schemes have proven to be the most accurate ones for problems which involve discontinuities in the flow 
as shock waves (see [19] for details). The latter are very frequently encountered in explosive events, since in these cases the flows can attain supersonic speeds. Shockcapturing schemes derive their accuracy from a discretization of the hydrodynamic equations which closely mimics the physics of compressible flows by making use of the Riemann problem, i.e. the dissolution of an arbitrary flow discontinuity into a set of simple waves (shocks, contact discontinuities and rarefaction waves). Suitably constructed Riemann problems at the interfaces between adjacent computational cells are solved within each time step, from which the complete solution of the system of conservation laws is constructed. This allows one to avoid the use of large amounts of artificial viscosity in order to obtain a well-behaved numerical scheme in the vicinity of shocks. One of the most accurate shock-capturing schemes, which has been widely applied in astrophysics, is the (direct Eulerian) PPM scheme of [3], a second order extension of Godunov's original (and rather diffusive) firstorder shock-capturing scheme [6]. In addition to its accurate treatment of shocks PPM includes a special detection and steepening algorithm to minimize numerical diffusion across contact discontinuities.

The superiority of shock-capturing schemes in computing compressible flows has been demonstrated e.g. in [19], and their performance for computing reactive astrophysical flows was studied in [4] and [13]. Fig. 1 shows a representative result from [4] in which PPM was compared to a number of older Eulerian schemes which were in wide-spread use until the mid 1980's. The figure shows the width of a contact discontinuity as a function of the number of zones that it has travelled across a numerical grid. Most Eulerian schemes tend to smear such fluid (and also composition) interfaces without limit, i.e. the width of the "discontinuity" tends to grow with time. Of all the schemes investigated, only PPM maintained a sharp resolution of the interface within two zones. Still however, numerical diffusion cannot be completely avoided in Eulerian calculations and its minimization necessitates an adequate spatial resolution in addition to an excellent advection scheme. This has led to the development of adaptive mesh refinement methods [1], which concentrate the computational effort in critical regions of the flow and thereby often allow for substantial savings in computer time.

\section{ADDITIONAL PHYSICS}

While the numerical problems encountered in solving the homogeneous part of the Euler equations are difficult to overcome, they represent only a part of the computational difficulties for a realistic simulation. The source terms, which are usually taken into account using the operator splitting technique [9], often require much more computer time than the solution of the hydrodynamic equations themselves. This holds, e.g. if large nuclear networks need to be evolved with the hydrodynamics or transport processes need to be taken into account (as e.g. neutrino transport in core collapse supernovae, see [15] and the references therein and A. Burrows, this volume). In some cases even phenomenological (sub-grid) models 
might have to be introduced. This is e.g. the case for turbulent combustion in thermonuclear supernovae, where a white dwarf is incinerated by a deflagration front whose propagation speed is impossible to compute in a direct simulation since this would require a resolution of the turbulent energy cascade down to the dissipation length scale [16]. Exacerbating the situation is the fact that stellar models, which serve as initial data for supernova simulations, might be affected by considerable uncertainties. In the absence of computational schemes and resources which allow for a consistent multidimensional treatment of stellar convection and rotation over stellar evolutionary time scales, one is forced to describe these phenomena by one-dimensional appoximations (see the contribution of N. Langer, this volume). Finally, uncertainties in nuclear reaction rates enter the calculations.

It is apparent that progress in only a single of the involved fields is not going to improve the accuracy of the desired nucleosynthetic yields considerably. In fact, a concerted effort in all areas appears to be required, since, as we will show in our example below, the different effects can conspire in falsifying the nucleosynthetic yields.

\section{CORE COLLAPSE SUPERNOVAE: A CASE STUDY}

Nucleosynthesis in core collapse supernovae is a good example for illustrating the aforementioned problems and we will start with a discussion of numerical diffusion using results of simple one-dimensional calculations. We subsequently address the complications introduced by convection in multidimensional calculations as well as by "additional physics", i.e. neutronization due to neutrino matter interactions. Finally we show how a multidimensional numerical failure, the so-called "oddeven-decoupling" phenomenon, an instability which appears to plague most shockcapturing schemes and whose effects have not yet been discussed extensively in the numerical astrophysics literature, can enhance neutronization by strengthening hydrodynamic convection and affect the nucleosythetic yields in multidimensional simulations.

\section{Nucleosynthesis in a $15 \mathrm{M}_{\odot}$ star (1D)}

In core collapse supernovae nucleosynthesis is triggered by a shock wave which forms after the collapse of the iron core of a massive star has proceeded to supranuclear densities. The shock, while initially powerful, stalls after a few milliseconds due to the energy losses from which it suffers while propagating through the outer iron core, but eventually ejects the outer stellar layers if heating by neutrinos from the collapsed core is able to overcompensate for the energy losses. In most nucleosynthesis calculations, however, these processes are not modelled in detail and instead a shock is initiated by simply depositing the typical observed supernova energy of $\sim 10^{51} \mathrm{erg}$ near the center of a presupernova model. 

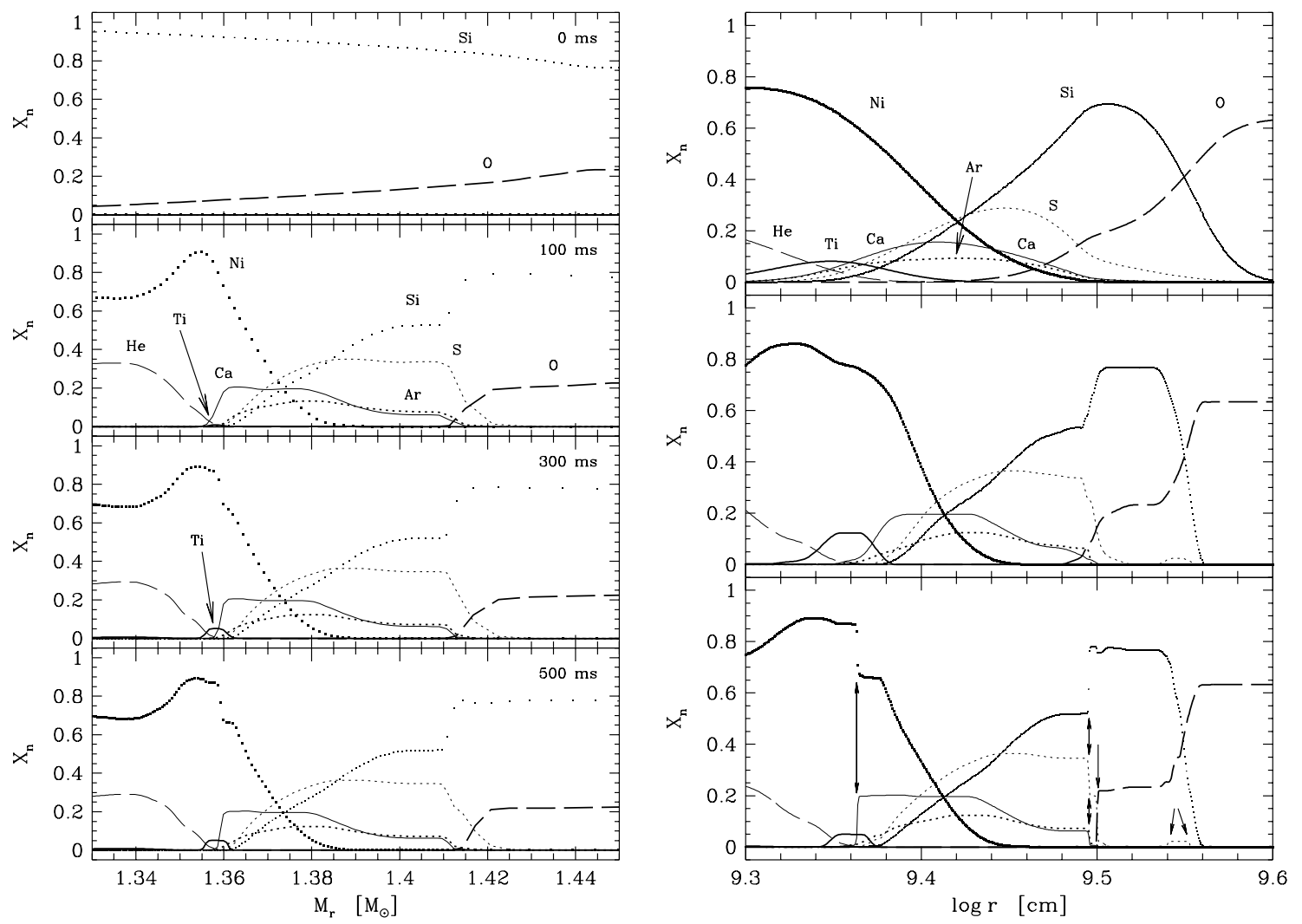

FIGURE 2. Left: Eulerian PPM calculation of explosive nucleosynthesis in the presupernova model of [20] using an $\alpha$-nucleus network and the Consistent Multifluid Advection scheme (CMA) (from [13]). Right: Comparison of Eulerian PPM results using: 1st order advection for nuclear species (top), the FMA advection scheme for multifluid flows of [4] (middle), and the CMA scheme of [13] (bottom). Note the decreasing amount of diffusion and the sharp interfaces obtained with CMA (from [13]).

In the left panels of Fig. 2 we show snapshots of the mass fractions from the first $500 \mathrm{~ms}$ of such a calculation, focusing on the silicon-rich layers just outside the iron core in which explosive nucleosynthesis takes place. Only $100 \mathrm{~ms}$ after the start of the calculations explosive silicon burning has frozen out and has left behind a significant abundance of ${ }^{56} \mathrm{Ni}$ as well as ${ }^{40} \mathrm{Ca},{ }^{36} \mathrm{Ar}$ and ${ }^{32} \mathrm{~S}$. Particularly noteworthy for the following discussion is the nucleus ${ }^{44} \mathrm{Ti}$. From one-dimensional Lagrangian nucleosynthesis calculations [17], [21] it is known that this isotope should be primarily synthesized in the innermost stellar layers which experience an $\alpha$-rich freezeout. However, in the present Eulerian calculation a significant abundance of ${ }^{44} \mathrm{Ti}$ has formed in zones with mass coordinates around $1.36 \mathrm{M}_{\odot}$ (marked with arrows in the left panels of Fig. 2), i.e. at the interface of the regions enriched in ${ }^{4} \mathrm{He}$ and ${ }^{40} \mathrm{Ca}$. This ${ }^{44} \mathrm{Ti}$ "bump" results from the reaction ${ }^{40} \mathrm{Ca}(\alpha, \gamma){ }^{44} \mathrm{Ti}$ and the amount 


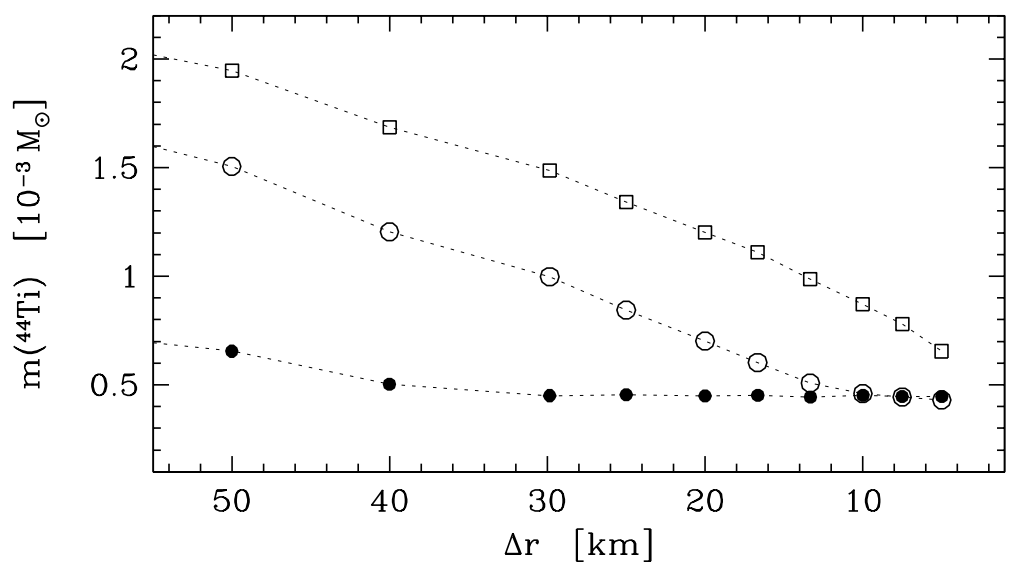

FIGURE 3. Final ${ }^{44} \mathrm{Ti}$ yield of the one-dimensional calculations shown in Fig. 2 as a function of radial resolution and different multifluid advection schemes. Top curve: 1st order species advection. Middle: FMA. Bottom: CMA (from [13]).

of ${ }^{44} \mathrm{Ti}$ thereby produced is very sensitive to numerical diffusion in this region. Consequently, the strength of the ${ }^{44} \mathrm{Ti}$ "bump" varies with the diffusivity of the numerical scheme which is used to advect the nuclear species. This is illustrated in the right panels of Fig. 2. The top right panel depicts results from a calculation with Godunov's first-order scheme. In this case, all mass fraction profiles are heavily smeared due to strong diffusion, as can be seen by a comparison to the middle right and bottom right panels which show results that were obtained with the FMA and CMA advection schemes of [4] and [13], respectively. Of all these schemes, CMA is the least diffusive since it is the only method which includes a detection and steepening algorithm for composition interfaces which was derived from PPM's original detection and steepening algorithm for contact discontinuities. Note the size of the ${ }^{44} \mathrm{Ti}$ bump for the three different runs. The more diffusive schemes produce much more ${ }^{44} \mathrm{Ti}$. This is also illustrated in Fig. 3 which summarizes how the ${ }^{44} \mathrm{Ti}$ yield depends on the adopted advection scheme and the spatial resolution. While the CMA results (bottom curve) are already converged for a resolution of $\Delta r=40 \mathrm{~km}$, FMA (middle curve) needs a resolution of about $10 \mathrm{~km}$. The first-order scheme (top curve) would need much finer zoning than $\Delta r=5 \mathrm{~km}$ to yield results of comparable quality. Note also that, if a diffusive advection scheme and coarse resolution are used, the errors might be as large as a factor of four!

It should be pointed out, however, that ${ }^{44} \mathrm{Ti}$ is a somewhat extreme (though very important) example. The (relative) errors due to numerical diffusion are usually smaller for the more abundant nuclei. This is illustrated in Fig. 4 which shows the dependence of the yields of different $\alpha$-nuclei on resolution in a 1D calculation from [8], in which no ad hoc energy deposition was adopted, but where the shock revival phase was followed in detail by including the effects of neutrino heating from a central light bulb neutrino source [7]. It can be seen from this figure that 


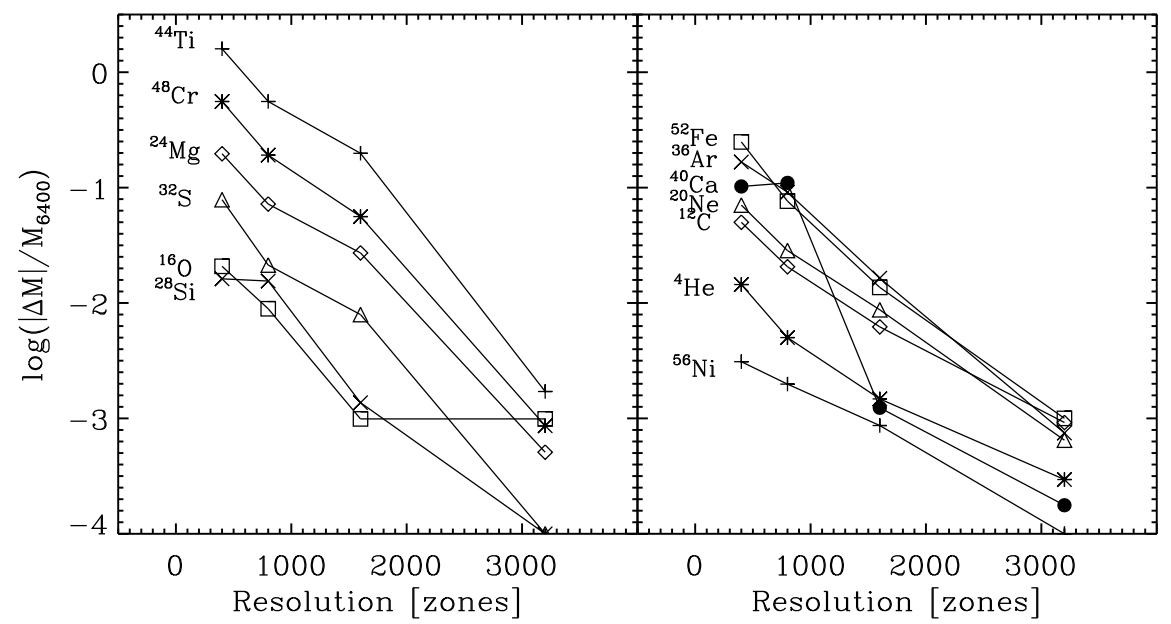

FIGURE 4. Accuracy of various elemental yields from one-dimensional shock-revival and explosive nucleosynthesis calculations in the post-bounce model of [2] (see also [20] for the presupernova model). The logarithm of the deviations of the elemental yields of various $\alpha$-nuclei as compared to an essentially converged 6400 zone calculation $(\Delta r \leq 5 \mathrm{~km})$ is displayed as a function of the radial resolution (in grid zones)(from [8]).

individual elemental yields are more accurate than the ${ }^{44} \mathrm{Ti}$ yield by more than an order of magnitude. However, if one is aiming at a numerical accuracy of about $1 \%$ for all yields, about 3000 radial zones are required for this calculation. This amount of spatial resolution makes accurate multidimensional simulations very expensive.

\section{Convection and ${ }^{56} \mathrm{Ni}$ synthesis in core collapse supernovae}

Neutrino matter interactions play a crucial role in the explosion of core collapse supernovae. They heat the matter behind the stalling shock and thereby trigger the explosion. On the other hand they determine the electron fraction per baryon, $Y_{\mathrm{e}}$, (or equivalently the ratio of protons to neutrons) in the ejecta and thus influence the nucleosynthetic yields. If the $Y_{\mathrm{e}}$ value of material that has been photodissociated by the shock is significantly reduced below 0.5 by neutrino/matter interactions, this matter will recombine mainly to neutron-rich nuclei after expanding and cooling. In that case nuclei with $Z=N$ like ${ }^{56} \mathrm{Ni}$ will not form in the ejecta. Fig. 5 which shows results of a two-dimensional simulation of shock revival illustrates this effect. In this calculation the luminosities of $\nu_{e}$ and $\bar{\nu}_{e}$ were such that $\bar{\nu}_{e}$ absorption on protons was favored against $\nu_{e}$ absorption on neutrons and thus the heated gas was neutronized [7]. The sharp division between this material which is visible in the bubbles behind the shock in Fig. 5 and the lepton-rich material farther out which has formed ${ }^{56} \mathrm{Ni}$ (the region enclosed by the white contour line in Fig. 5) is clearly visible. The negative entropy gradient that the heating has imprinted on the layers between the radius of maximum neutrino heating (close to the center) and the shock farther out, has also led to strong convective motions: bubbles of heated 


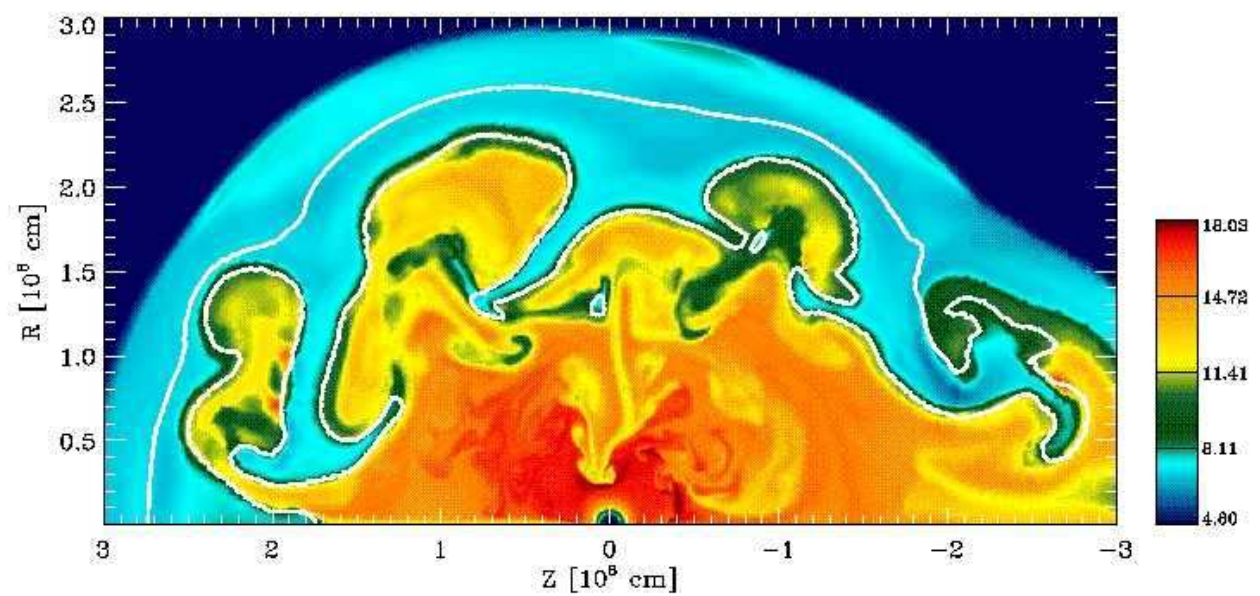

FIGURE 5. Distribution of the entropy (in units of $k_{B} /$ nucl.) $320 \mathrm{~ms}$ after core bounce in a two-dimensional core collapse supernova simulation of a $15 \mathrm{M}_{\odot}$ star. The white contour line encloses the region in which the ${ }^{56} \mathrm{Ni}$ mass fraction exceeds $20 \%$ (from [8]).

deleptonized gas rise toward the shock while lower entropy flux tubes transport lepton-rich matter to deeper layers where it interacts with the neutrino fluxes much more efficiently. This interplay of convection and deleptonization is crucial for the ${ }^{56} \mathrm{Ni}$ yield. The shock is only able to heat a certain amount of lepton-rich material to temperatures in excess of the $5 \times 10^{9} \mathrm{~K}$ which are required for ${ }^{56} \mathrm{Ni}$ synthesis. If convection is strong enough to advect significant amounts of this matter close to the neutron star, where the gas will experience deleptonization, the ${ }^{56} \mathrm{Ni}$ yield will be lower than in a model with no or weak convection.

\section{Multidimensional numerical failures: Odd-even decoupling}

Quirk [14] has reported on a subtle flaw in a number of shock capturing schemes which becomes evident when calculating multidimensional flows with strong, gridaligned shocks. He has dubbed this failure the "odd-even decoupling" phenomenon. The problem shows up only if a sufficiently strong shock is either fully or nearly aligned with one of the coordinate directions of the grid, and if, in addition, the flow is slightly perturbed. This can be due to either perturbations intentionally introduced in order to study physical instabilities, as it is done in all studies of convection in supernovae, or due to perturbations caused by other flow features. Many Riemann solvers show the tendency to allow these perturbations to grow without limit along the shock surface, thus triggering a strong rippling of the shock front as well as the post shock state. In supernova simulations these perturbations, whose amplitudes can exceed those of the seed perturbations by several orders of magnitude, enhance the growth of hydrodynamic instabilities. In case of neutrino driven convection they lead to large-scale overturn and angular wavelengths of convective bubbles which are significantly larger than in a "clean" calculation. This 

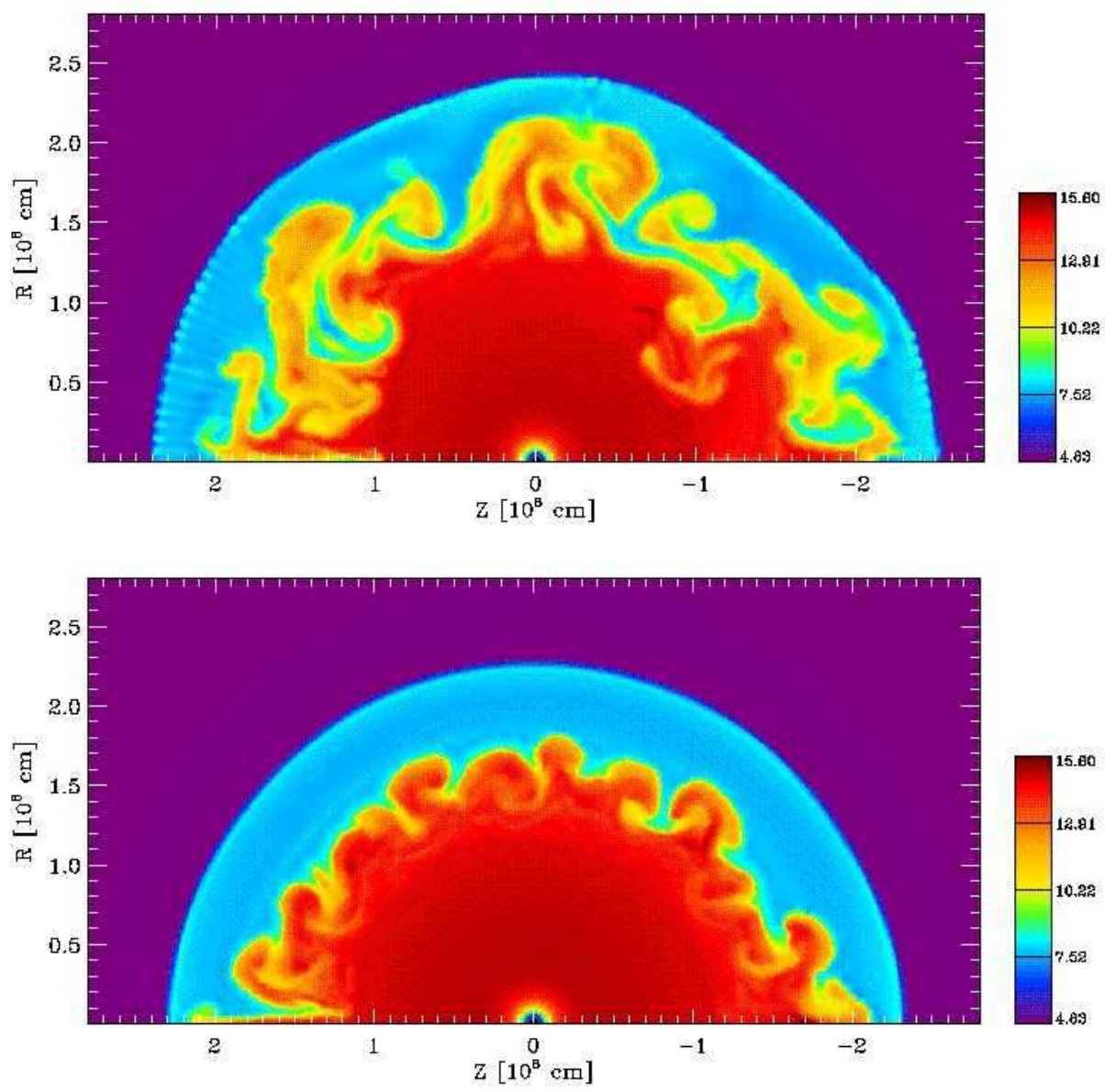

FIGURE 6. Top: Entropy distribution $208 \mathrm{~ms}$ after core bounce (in units of $k_{B} /$ nucl.) in a two-dimensional supernova model showing odd-even decoupling. Bottom: Entropy distribution for an equivalent calculation in which odd-even decoupling has been suppressed (from [8]).

artificial enhancement of convection is demonstrated in Fig. 6 where the entropy distribution of a simulation exhibiting odd-even decoupling (top panel) is compared to one in which the numerical failure has been cured (bottom panel). A modification of PPM's original dissipation algorithms [3] was necessary for this purpose. Alternatively the hybrid Riemann solver method of [14] might be used. Note that the calculations have been carried out in spherical coordinates $(r, \theta)$ so that the (initially spherical) shock wave was fully aligned with the grid. However, cylindrical coordinates which have been used for plotting are indicated in the figures. The difference in the final ${ }^{56} \mathrm{Ni}$ yield for these two simulations was about $40 \%$, the calculation not exhibiting odd-even decoupling showing the larger yield, as expected from our discussion in the previous section. This demonstrates that due to the strong coupling of neutrino physics and convection, the ${ }^{56} \mathrm{Ni}$ yield in multidimensional calculations is much more difficult to calculate correctly than in one spatial dimension. Therefore the error of at most a few percent which can be deduced 
for the latter case from Fig. 4 can be deceptive. The results shown in Fig. 6 also suggest that numerical noise, whatever its origin is, leads to a grossly overestimated efficiency of convection. Thus, schemes which are known to suffer from this problem (like SPH) do not appear to be suited for nucleosynthesis calculations in core collapse supernovae.

\section{CONCLUSIONS}

Realistic nucleosynthesis calculations in astrophysical contexts represent a challenge in many respects. The difficulties involve the numerical treatment of multidimensional hydrodynamic advection, complex physics in addition to hydrodynamics and burning, disparate length and time scales, realistic initial conditions and uncertainties in reaction rates. It is our conviction, that substantial efforts are required in each of these fields in order to obtain reliable yields in multidimensional nucleosynthesis calculations.

\section{REFERENCES}

1. Berger, M., and Colella, P., Jour. of Comp. Phys., 82, 64 (1989).

2. Bruenn S. W., Nuclear Physics in the Universe, Bristol: IOP, 1993, p.31

3. Colella, P., and Woodward, P. R., Jour. of Comp. Phys., 54, 174 (1984).

4. Fryxell B., Müller E., and Arnett W. D., MPA Preprint, 449, 1 (1989).

5. Fryxell B., Müller E., and Arnett W. D., ApJ, 367, 619 (1991).

6. Godunov S. K., Mat. Sb., 47, 271 (1959).

7. Janka H.-T., and Müller E., A\&A, 306, 167 (1996).

8. Kifonidis K., PhD thesis, Technische Universität München, (2000).

9. LeVeque R. J., Computational Methods for Astrophysical Fluid Flow, Berlin: Springer, 1998, pp. 84-101

10. Müller E., Computational Methods for Astrophysical Fluid Flow, Berlin: Springer, 1998, pp. 463-480

11. Oran, E. S., and Boris, J. P., Numerical Simulation of Reactive Flow, New York: Elsevier, 1987, ch. 10, pp. 358-394.

12. Pen, U.-L., ApJS, 115, 19 (1998).

13. Plewa T., and Müller E., A\&SA, 342, 179 (1999).

14. Quirk J. J., Int. J. Num. Meth. Fluids, 18, 555 (1994).

15. Rampp M., and Janka H.-T., ApJ, 539, L33 (2000).

16. Reinecke M., Hillebrandt W., and Niemeyer J. C., A $\& A$ A, 347, 739 (1999).

17. Thielemann F.-K., Nomoto K. I., and Hashimoto M., ApJ, 460, 408 (1996).

18. Woodward, P. R., ApJ, 207, 484 (1976).

19. Woodward, P. R., and Colella, P., Jour. of Comp. Phys., 54, 115 (1984).

20. Woosley S. E., Pinto P. A., and Ensman L., ApJ, 324, 466 (1988).

21. Woosley S. E., and Weaver T. A., ApJS, 101, 181 (1995). 